\title{
A New No-Equilibrium Chaotic System and Its Topological Horseshoe Chaos
}

\author{
Chunmei Wang, ${ }^{1}$ Chunhua Hu, ${ }^{2}$ Jingwei Han, ${ }^{3}$ and Shijian Cang ${ }^{4,5}$ \\ ${ }^{1}$ Department of Information Engineering, Binzhou University, Binzhou 256600, China \\ ${ }^{2}$ Department of Electrical Engineering, Binzhou University, Binzhou 256600, China \\ ${ }^{3}$ College of Aeronautical Engineering, Binzhou University, Binzhou 256600, China \\ ${ }^{4}$ Department of Product Design, Tianjin University of Science and Technology, Tianjin 300457, China \\ ${ }^{5}$ School of Electrical Engineering and Automation, Tianjin University, Tianjin 300072, China \\ Correspondence should be addressed to Shijian Cang; sj.cang@gmail.com
}

Received 13 September 2016; Revised 11 November 2016; Accepted 6 December 2016

Academic Editor: Xavier Leoncini

Copyright (C) 2016 Chunmei Wang et al. This is an open access article distributed under the Creative Commons Attribution License, which permits unrestricted use, distribution, and reproduction in any medium, provided the original work is properly cited.

\begin{abstract}
A new no-equilibrium chaotic system is reported in this paper. Numerical simulation techniques, including phase portraits and Lyapunov exponents, are used to investigate its basic dynamical behavior. To confirm the chaotic behavior of this system, the existence of topological horseshoe is proven via the Poincaré map and topological horseshoe theory.
\end{abstract}

\section{Introduction}

Since Lorenz found an atmosphere dynamical model which can generate butterfly-shaped chaotic attractor in 1963 [1], chaos theory in the past five decades has attracted a lot of attention and hence triggered the emergence of a huge literature in this area. Since then, many kinds of chaotic or hyperchaotic systems governed by nonlinear ordinary differential equations (ODEs), including autonomous and nonautonomous chaotic systems [2-4], continuous and discrete chaotic systems [5-7], integer-order and fractionalorder chaotic systems $[1,2,7,8]$, and chaotic systems with self-excited attractor and hidden attractor [9-11], were developed, and continuous chaotic systems governed by nonlinear partial differential equations (PDEs) [12-14] were also investigated.

To our knowledge, we summarize four criteria for the existence of chaos in the investigation of dynamical systems. The first one is the well-known Lyapunov exponents [15]. If there is at least one positive Lyapunov exponent in a dynamical system, the dynamics of this system is chaotic. The second one is Sil'nikov's criterion for the existence of chaos [16, 17]. The main steps are as follows: (1) calculate equilibrium points of a dynamical system; (2) find a homoclinic or heteroclinic orbit connecting equilibrium points by using the undetermined coefficient method; and (3) prove the convergence of the homoclinic or heteroclinic orbit series expansion obtained before. If the convergence can be proved, horseshoe chaos occurs. The third one is Melnikov's criterion which is a powerful approximate tool for investigating chaos occurrence in near Hamiltonian systems and has been successfully applied to the analysis of chaos in smooth systems by calculating the distance between the stable and unstable manifold [18]. For dynamical systems, when the stable and unstable manifolds of their fixed points in the Poincaré map intersect transversely for sufficiently small parameter, there exists chaos in the sense of Smale horseshoe. The last one is the topological horseshoes theory which is based on the geometry of continuous maps on some subsets of interest in state space [19-23]. It is more applicable for computerassisted verifications for the existence of chaotic behavior in dynamical systems in theory. A comparative analysis of these methods shows that the calculation of Lyapunov exponents and the topological horseshoes theory can be widely applied, but the Sil'nikov criterion is suitable for these systems where there is a homoclinic or heteroclinic orbit. Obviously, the Sil'nikov criterion cannot be used in no-equilibrium systems.

Very recently, hidden attractor in dynamical systems has been an important research topic because it has properties different from self-excited attractor. An attractor is called the 
hidden attractor if its basin of attraction does not intersect with small neighborhoods of the unstable fixed point; that is, the basins of attraction of the hidden attractors do not touch unstable fixed points and are located far away from such points [9]. The hidden attractors have been observed in these systems without fixed points, with no unstable fixed points, or with one stable fixed point, which motivate further construction and study of various artificial chaotic systems without equilibria. So far, hidden chaotic attractor has not been studied by the topological horseshoe theory. The main contribution of this research is to propose a new no-equilibrium system and verify the existence of its chaotic behavior by topological horseshoes theory. The proposed system is an artificial chaotic system having hidden attractor and has simple structure. The objective of this study is that we try to use the topological horseshoe theory to verify the existence of chaotic behavior. The rest of this paper is organized as follows. In Section 2, we introduce a no-equilibrium chaotic system and analyze its basic dynamics. In Section 3, we present rigorous arguments on existence of chaos in the new no-equilibrium system via topological horseshoe theory and computer computations. The conclusion is presented in the last section.

\section{The Proposed No-Equilibrium System}

2.1. Mathematical Model. Consider the following threedimensional dynamical system:

$$
\begin{aligned}
& \dot{x}=a y, \\
& \dot{y}=-b x-c y+y z, \\
& \dot{z}=-y^{2}+k,
\end{aligned}
$$

where $x, y$, and $z$ make up the system variables, and the constant term $k$ is an external DC excitation, while $a, b, c \in$ $\mathbb{R}^{+}$are the system's parameters. When $a=2, b=5, c=10$, and $k=15$, system (1) can generate a chaotic attractor under the initial condition $\left(x_{0}, y_{0}, z_{0}\right)=(-2,1,1)$, as shown in Figure 1.

2.2. Basic Dynamics Analysis. Consider the symmetry and invariance; it is easy to get the invariance of system (1) under the coordinate transformation $(x, y, z) \rightarrow(-x,-y, z)$; namely, system (1) has rotation symmetry around the $z$-axis. We note that the divergence of flow of system (1) is

$$
\nabla V=\frac{\partial \dot{x}}{\partial x}+\frac{\partial \dot{y}}{\partial y}+\frac{\partial \dot{z}}{\partial z}=-c+z
$$

Since $\nabla V$ is not always smaller than zero, it is hard to directly determine the dissipativity of system (1), which is very special through a comparison of the general chaotic systems. Solving $\dot{x}=\dot{y}=\dot{z}=0$, we cannot obtain exact real solution. Therefore, system (1) is a no-equilibrium system and the chaotic attractor shown in Figure 1 is a hidden attractor [24-27].

2.3. Numerical Analysis. For a three-dimensional dynamical system, we know that it has distinct features in its Lyapunov exponents. If there is one positive Lyapunov exponent, the dynamics of this system is chaotic. If we assume that the largest Lyapunov exponent of system (1) is $L_{\max }$, then by calculation, we will find that system (1) enters into chaotic states under the condition of different parameter values. The largest Lyapunov exponent spectrum of system (1) with respect to $a$ is depicted in Figure 2. As $a=2$, the largest Lyapunov exponent $L_{\max }$ is 0.1273 , so we have reasons to believe that system (1) is chaotic in this case.

\section{Horseshoe Chaos in the New System}

3.1. Reviews of Topological Horseshoe Theory. In this section, we firstly recall a result on horseshoes theory developed in $[22,23]$. The dynamics generated by Smale's horseshoe is very important as a basic mechanism that indicates clearly the complexity of the chaotic behavior. In order to describe clearly the chaotic attractor shown in Figure 1, the topological horseshoe is introduced in this section.

Let $S_{m}=\{0,1, \ldots, m-1\}$ be the set of nonnegative successive integers from 0 to $m-1$. Let $\Sigma_{m}$ be a metric space, which is compact, totally disconnected, and perfect. A set having the three properties is often defined as a Cantor set; such a Cantor set frequently appears in characterization of complex structure of invariant set in a chaotic dynamical system. An $m$-shift map $\sigma: \Sigma_{m} \rightarrow \Sigma_{m}$ is defined as follows:

$$
\sigma(s)_{i}=s_{i+1},
$$

where $s_{i} \in S_{m}$, with $i=1,2, \ldots, m$. Then we get $\sigma\left(\Sigma_{m}\right)=\Sigma_{m}$ and $\sigma$ is continuous, and the $m$-shift map $\sigma$ as a dynamical system defined on $\Sigma_{m}$ has the following properties: (1) $\sigma$ has a countable infinity of periodic orbits consisting of orbits of all periods; (2) $\sigma$ has an uncountable infinity of nonperiodic orbits; and (3) $\sigma$ has a dense orbit [23].

Let $X$ be a metric space, $D$ be a compact subset of $X$, and $f: D \rightarrow X$ be a map satisfying the assumption that there exist $m$ mutually disjoint compact subsets $D_{1}, D_{2}, \ldots$, and $D_{m}$ in $D$, and the restriction of $f$ to each $D_{i}$ is continuous; namely, $f: D_{i} \rightarrow X$ is continuous map, where $i=1,2, \ldots, m$.

Definition 1. Let $\gamma$ be a compact subset of $D$, such that, for each $i \in\{1,2, \ldots, m\}, \gamma_{i}=\gamma \cap D_{i}$ is nonempty and compact; then $\gamma$ is called a connection with respect to $D_{1}, D_{2}, \ldots$, and $D_{m}$. Let $F$ be a family of connections $\gamma s$ with respect to $D_{1}$, $D_{2}, \ldots$, and $D_{m}$ satisfying the following property: $\gamma \in F \Rightarrow$ $f\left(\gamma_{i}\right) \in F$. Then $F$ is said to be a $f$-connected family with respect to $D_{1}, D_{2}, \ldots$, and $D_{m}$.

Next, we recall the semiconjugacy in terms of a continuous map and the shift map $\sigma$, which is conventionally defined as follows.

Definition 2. Let $X$ be a metric space. Consider a continuous map $f: X \rightarrow X$, and let $\Lambda$ be a compact invariant set of $f$. If there exists a continuous and onto map ent : $\Lambda \rightarrow \Sigma_{m}$ such that ent $\circ f=\sigma \circ$ ent, then $f$ is said to be semiconjugate to $\sigma$.

Theorem 3. Suppose that there exists a $f$-connected family $F$ with respect to disjointed compact subsets $D_{1}, D_{2}, \ldots$, and $D_{m}$. 


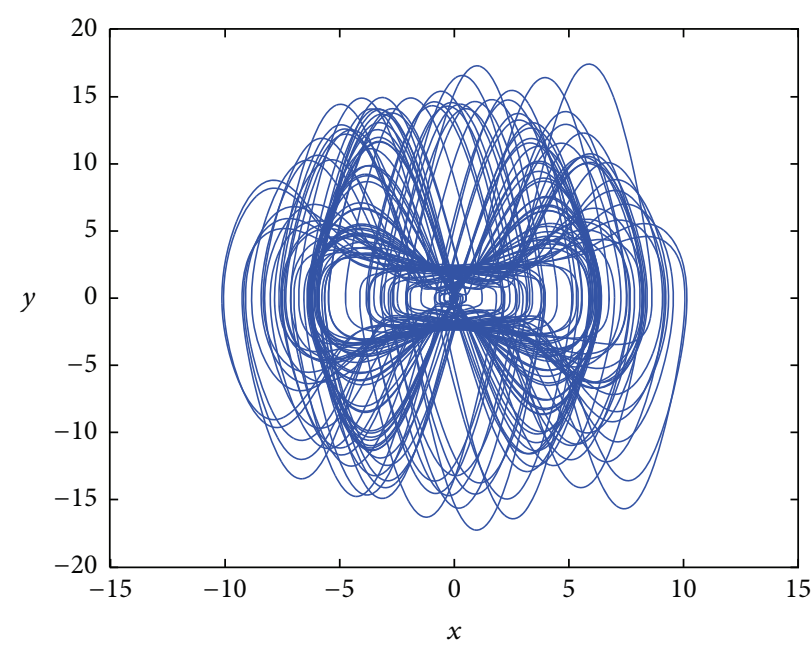

(a)

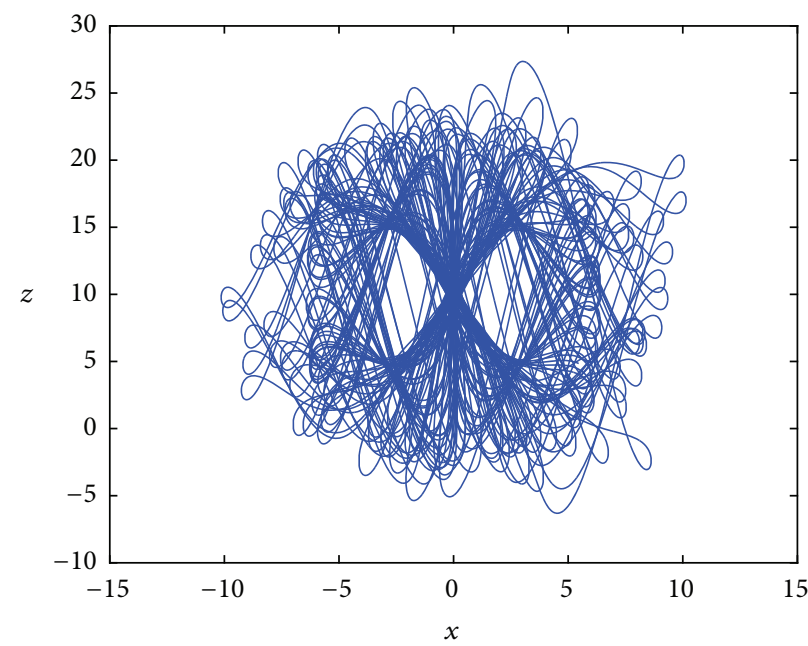

(b)

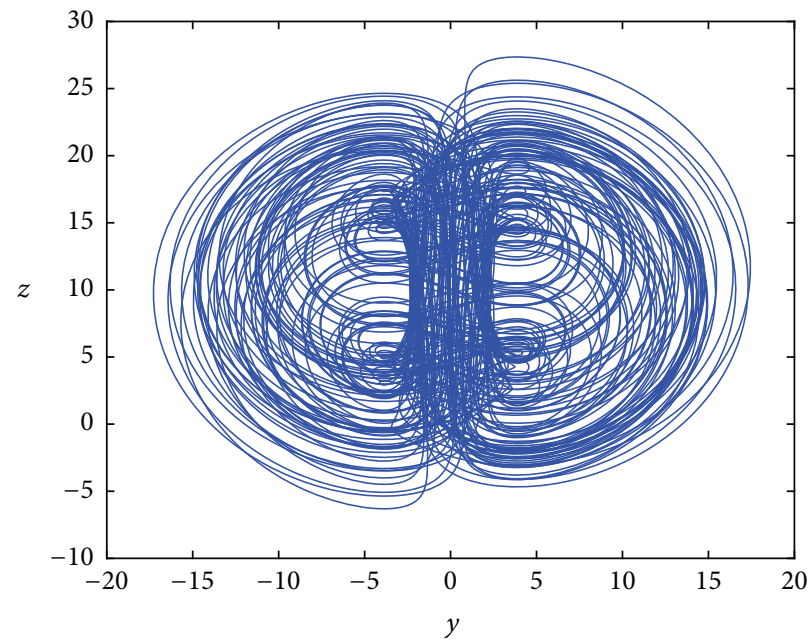

(c)

FIGURE 1: Chaotic attractor of system (1) with $a=2, b=5, c=10$, and $k=15$ and initial condition $\left(x_{0}, y_{0}, z_{0}\right)=(-2,1,1):(\mathrm{a}) x-y$ plane; (b) $x-z$ plane; (c) $y-z$ plane.

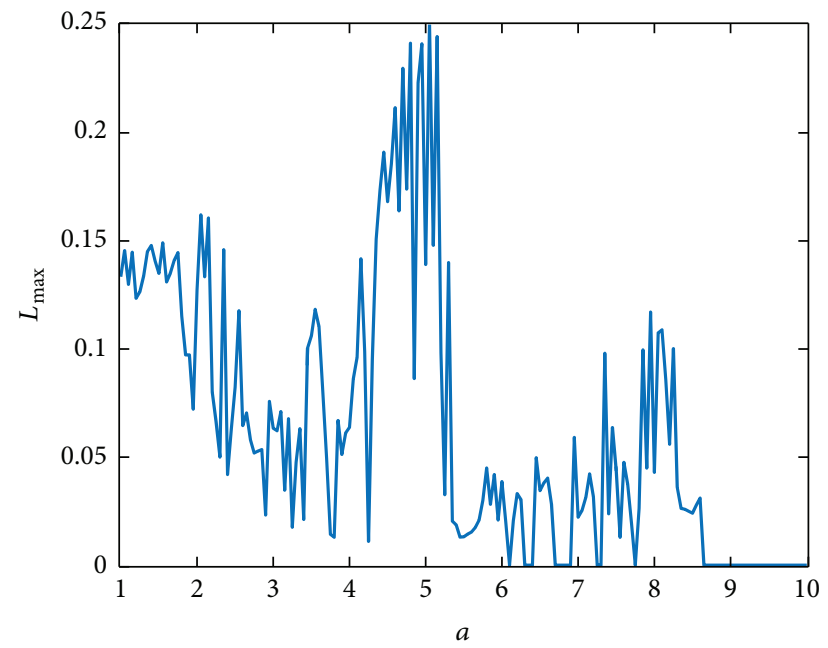

FIGURE 2: The largest Lyapunov exponent spectrum of system (1) versus $a$.
Then there exists a compact invariant set $\Lambda \subset D$, such that $f \mid \Lambda$ is semiconjugate to $m$-shift.

Lemma 4. Let $X$ be a compact metric space, and $f: X \rightarrow X$ be a continuous map. If there exists an invariant set $\Lambda \subset X$ such that $f \mid \Lambda$ is semiconjugate to the $m$-shift $\sigma$, then

$$
\text { ent }(f) \geq \operatorname{ent}(\sigma)=\log m \text {, }
$$

where $\operatorname{ent}(f)$ denotes the entropy of map $f$. In addition, for every positive integer $k$, ent $\left(f^{k}\right)=k \cdot \operatorname{ent}(f)$. When $m>1$, the dynamics generated by the shift map $\sigma$ has a positive topological entropy and, therefore, is sensitive to initial conditions, which means that $f$ must be chaotic.

3.2. Topological Horseshoe Analysis of the Proposed Chaotic System. In this section, a rigorous verification of chaos in the chaotic system (1) by combining the topological horseshoe theory with a computer-assisted method of Poincaré maps 


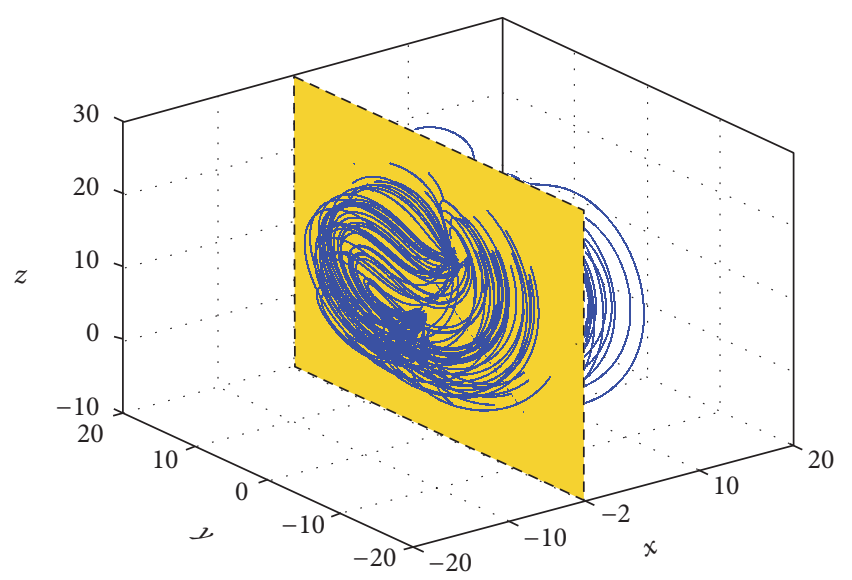

FIgURE 3: Phase portrait of system (1) and the cross section $\Gamma$ projected onto $x-y-z$ space.

will be presented. For this purpose, we will utilize the technique of cross section and the corresponding Poincaré maps. Denote by $\varphi\left(X_{0}, t\right)$ the flow of system (1) with initial condition $X_{0}=\left(x_{0}, y_{0}, z_{0}\right)=(-2,1,1)$, that is, $\varphi\left(X_{0}, 0\right)=$ $X_{0}$. As shown in Figure 3, we choose a 3D cross section $\Gamma=\left\{(x, y, z) \in \mathbb{R}^{3} \mid x=-2\right\}$.

Now in the plane $\Gamma=\left\{(x, y, z) \in \mathbb{R}^{3} \mid x=-2\right\}$, after many trial-and-error numerical simulations, we can take a rectangular $Q=|A B C D|$ with its four vertices being $A=[-2,0,-20], B=[-2,25,-20], C=[-2,25,40]$, and $D=[-2,0,40]$ as our cross section and define the corresponding Poincaré map $H: Q \rightarrow \Gamma$. For every point $m(x, y, z)$ in this rectangle, the point $H(m)$ is defined to be the intersection point of $\Gamma$ with the flow $\varphi(m, t)$ with initial condition $m(x, y, z)$ when first returning to the plane $\Gamma$. In order to find horseshoe, we consider four compositions of the map $\bar{H}=H^{4}$; namely, $\bar{H}: Q \rightarrow \Gamma$.

Proposition 5. The Poincaré map H corresponding to the cross section $Q=|A B C D|$ has the property that there exists a closed invariant set $\Lambda \subset Q$ for which $\bar{H} \mid \Lambda$ (i.e., $H^{4} \mid \Lambda$ ) is semiconjugate to the 2-shift map, and ent $(\bar{H}) \geq \log 2>0$, so the Poincaré map $H$ of system (1) with $a=2, b=5, c=10$, $k=15$ and initial condition $\left(x_{0}, y_{0}, z_{0}\right)=(-2,1,1)$ is chaotic.

Proof. To prove this statement, we take two subsets $D_{1}$ and $D_{2}$ of $H$ shown in Figure 4, with the vertexes of $D_{1}$ being $[-2,4.757964974,5.051336502]$, [ $-2,4.766024338$, $5.054745948],[-2,4.765806517,5.004091334]$, and $[-2$, $4.757311513,5.002143080]$ and the vertexes of $D_{2}$ being [ -2 , 4.843731848, 5.044882910], [-2, 4.845637779, 5.044517612], $[-2,4.845583324,5.008718438]$, and $[-2,4.843568483$, 5.008231375].

Let $D_{1}^{1}$ and $D_{1}^{2}$ denote the left and right sides of $D_{1}$, respectively, and $D_{2}^{1}$ and $D_{2}^{2}$ denote the left and right sides of $D_{2}$, respectively. Numerical simulations show that the images $\bar{H}\left(D_{1}\right)$ and $\bar{H}\left(D_{2}\right)$ lie wholly across the quadrangle $D_{1}$ and $D_{2}$, and $\bar{H}\left(D_{1}^{2}\right)$ and $\bar{H}\left(D_{2}^{2}\right)$ lie on the left side of $D_{1}$ and $D_{2}$,

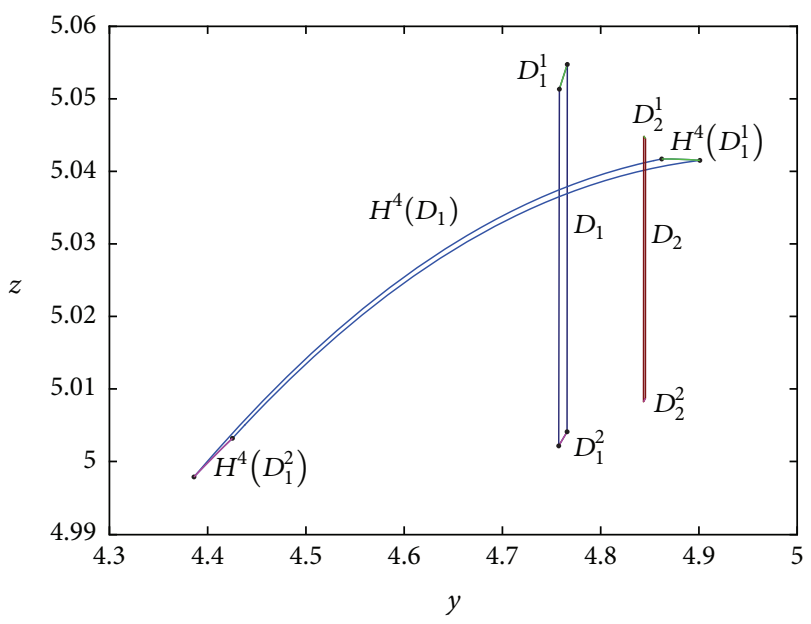

Figure 4: $\bar{H}\left(D_{1}\right)$ wholly across the quadrangle $D_{1}$ and $D_{2}$.

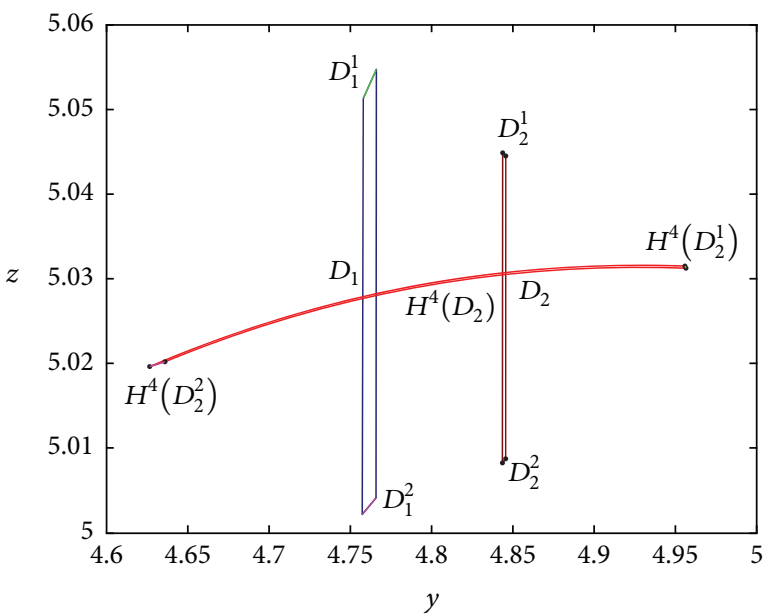

FIGURE 5: $\bar{H}\left(D_{2}\right)$ wholly across the quadrangle $D_{1}$ and $D_{2}$.

and $\bar{H}\left(D_{1}^{1}\right)$ and $\bar{H}\left(D_{2}^{1}\right)$ lie on the right side of $D_{1}$ and $D_{2}$, which are shown in Figures 4 and 5. In view of the definition of $m$-shift map, there exists an $H$-connected family with respect to these two subsets $D_{1}$ and $D_{2}$. Therefore, according to Theorem 3, it can be concluded that the Poincaré map $H$ is semiconjugated to a 2 -shift map.

Now let $F$ be the family of connections with its element $\gamma$ satisfying that $\gamma \subset Q$ is a path and $\gamma$ goes through $D_{1}$ with intersection points in $D_{1}$ and $D_{2}$ and goes through $D_{2}$ with intersection points in $D_{1}$ and $D_{2}$; then from the above arguments it is easy to see that, for every $\gamma_{i}=\gamma \cap D_{i}(i=1,2)$, we have $\bar{H}\left(\gamma_{i}\right) \in F$. Now it follows from horseshoe lemma that there exists a compact invariant set $\Lambda \subset Q$, such that $\bar{H} \mid \Lambda$ is semiconjugate to 2 -shift map. In view of Lemma 4 , it is easy to see that $\operatorname{ent}(H)=\operatorname{ent}(\bar{H}) / 8 \geq \log 2 / 8$, which means that the map $H$ is chaotic. The chaos of the Poincaré map $H$ implies that chaos of the original system (1). Thus we prove that system (1) is chaotic for the given parameters. 


\section{Conclusion}

In this paper, we proposed a new no-equilibrium system which can generate chaotic flow for the given parameters. Numerical simulation techniques, including phase portraits and Lyapunov exponents, illustrate its chaotic behavior. Moreover, numerical simulations show that the existence of horseshoe chaos in the proposed system is proven by means of topological horseshoe theory. The essence of the arguments is to choose a cross section and study the dynamics of the corresponding Poincaré map to which the topological horseshoe theory can apply. The horseshoe chaos in the Poincaré map shows that the proposed system does exhibit chaotic behavior.

\section{Competing Interests}

The authors declare that there is no conflict of interests regarding the publication of this paper.

\section{Acknowledgments}

This work was supported in part by the Shandong Natural Science Foundation of China under Grant no. ZR2014FQ019 and the Science Foundation of Binzhou University of China under Grant nos. BZXYG1618 and BZXYG1615.

\section{References}

[1] E. N. Lorenz, "Deterministic nonperiodic flow," Journal of the Atmospheric Sciences, vol. 20, no. 2, pp. 130-141, 1963.

[2] G. Chen and T. Ueta, "Yet another chaotic attractor," International Journal of Bifurcation and Chaos, vol. 9, no. 7, pp. 14651466, 1999.

[3] W. Zhang and W. L. Hao, "Multi-pulse chaotic dynamics of six-dimensional non-autonomous nonlinear system for a composite laminated piezoelectric rectangular plate," Nonlinear Dynamics, vol. 73, no. 1-2, pp. 1005-1033, 2013.

[4] S. Cang, A. Wu, Z. Wang, W. Xue, and Z. Chen, "Birth of one-to-four-wing chaotic attractors in a class of simplest three-dimensional continuous memristive systems," Nonlinear Dynamics, vol. 83, no. 4, pp. 1987-2001, 2016.

[5] N. Lazaryan and H. Sedaghat, "Periodic and chaotic orbits of a discrete rational system," Discrete Dynamics in Nature and Society, vol. 2015, Article ID 519598, 8 pages, 2015.

[6] D. Arroyo, G. Alvarez, S. Li, C. Li, and J. Nunez, "Cryptanalysis of a discrete-time synchronous chaotic encryption system," Physics Letters A, vol. 372, no. 7, pp. 1034-1039, 2008.

[7] S. Cang, Z. Wang, Z. Chen, and H. Jia, "Analytical and numerical investigation of a new Lorenz-like chaotic attractor with compound structures," Nonlinear Dynamics, vol. 75, no. 4, pp. 745-760, 2014.

[8] S. Cang, Z. Chen, Z. Wang, and H. Jia, "Projective synchronisation of fractional-order memristive systems with different structures based on active control method," International Journal of Sensor Networks, vol. 14, no. 2, pp. 102-108, 2013.

[9] D. Dudkowski, S. Jafari, T. Kapitaniak, N. V. Kuznetsov, G. A. Leonov, and A. Prasad, "Hidden attractors in dynamical systems," Physics Reports-Review Section of Physics Letters, vol. 637, pp. 1-50, 2016.
[10] G. A. Leonov and N. V. Kuznetsov, "Hidden attractors in dynamical systems. From hidden oscillations in HilbertKolmogorov, Aizerman, and KALman problems to hidden chaotic attractor in Chua circuits," International Journal of Bifurcation and Chaos in Applied Sciences and Engineering, vol. 23, no. 1, Article ID 1330002, 69 pages, 2013.

[11] G. A. Leonov, N. V. Kuznetsov, and V. I. Vagaitsev, "Localization of hidden Chua's attractors," Physics Letters A, vol. 375, no. 23, pp. 2230-2233, 2011.

[12] M. Khan, T. Shah, and M. A. Gondal, "An efficient technique for the construction of substitution box with chaotic partial differential equation," Nonlinear Dynamics, vol. 73, no. 3, pp. 1795-1801, 2013.

[13] M. P. Pettersson, G. Iaccarino, and J. Nordstrom, Polynomial Chaos Methods for Hyperbolic Partial Differential Equations, Mathematical Engineering, Springer, 2015.

[14] Y. Zhang, D. Xiao, Y. Shu, and J. Li, "A novel image encryption scheme based on a linear hyperbolic chaotic system of partial differential equations," Signal Processing: Image Communication, vol. 28, no. 3, pp. 292-300, 2013.

[15] A. Wolf, J. B. Swift, H. L. Swinney, and J. A. Vastano, "Determining Lyapunov exponents from a time series," Physica D. Nonlinear Phenomena, vol. 16, no. 3, pp. 285-317, 1985.

[16] L. Zhou and F. Chen, "Sil'nikov chaos of the Liu system," Chaos, vol. 18, no. 1, Article ID 013113, 2008.

[17] T. Zhou, G. Chen, and S. Čelikovský, "Ši’lnikov chaos in the generalized Lorenz canonical form of dynamical systems," Nonlinear Dynamics, vol. 39, no. 4, pp. 319-334, 2005.

[18] W. Xu, J. Feng, and H. Rong, "Melnikov's method for a general nonlinear vibro-impact oscillator," Nonlinear Analysis, Theory, Methods \& Applications, vol. 71, no. 1-2, pp. 418-426, 2009.

[19] G. Cian, "Some remarks on topological horseshoes and applications," Nonlinear Analysis: Real World Applications, vol. 16, pp. 74-89, 2014.

[20] Q. Li and X.-S. Yang, "A simple method for finding topological horseshoes," International Journal of Bifurcation and Chaos in Applied Sciences and Engineering, vol. 20, no. 2, pp. 467-478, 2010.

[21] X.-S. Yang, "Topological horseshoes and computer assisted verification of chaotic dynamics," International Journal of Bifurcation and Chaos in Applied Sciences and Engineering, vol. 19, no. 4, pp. 1127-1145, 2009.

[22] X.-S. Yang and Y. Tang, "Horseshoes in piecewise continuous maps," Chaos, Solitons \& Fractals, vol. 19, no. 4, pp. 841-845, 2004.

[23] Q. Li and X.-S. Yang, "Chaotic dynamics in a class of three dimensional Glass networks," Chaos, vol. 16, no. 3, Article ID 033101, 2006

[24] V. Pham, S. Vaidyanathan, C. Volos, S. Jafari, N. Kuznetsov, and T. Hoang, "A novel memristive time-delay chaotic system without equilibrium points," The European Physical Journal Special Topics, vol. 225, no. 1, pp. 127-136, 2016.

[25] A. Akgul, H. Calgan, I. Koyuncu, I. Pehlivan, and A. Istanbullu, "Chaos-based engineering applications with a 3D chaotic system without equilibrium points," Nonlinear Dynamics, vol. 84, no. 2, pp. 481-495, 2016.

[26] D. Cafagna and G. Grassi, "Chaos in a new fractional-order system without equilibrium points," Communications in Nonlinear Science and Numerical Simulation, vol. 19, no. 9, pp. 2919-2927, 2014. 
[27] Z. Wang, S. Cang, E. O. Ochola, and Y. Sun, "A hyperchaotic system without equilibrium," Nonlinear Dynamics, vol. 69, no. 1-2, pp. 531-537, 2012. 


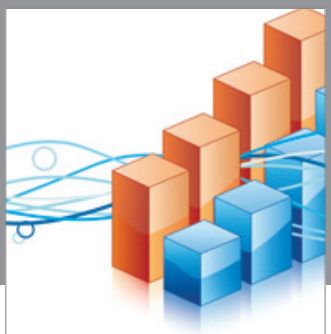

Advances in

Operations Research

vatem alat4

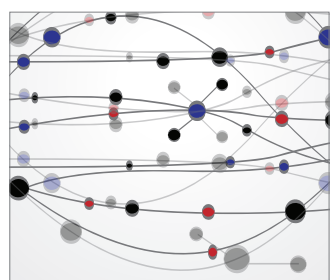

\section{The Scientific} World Journal
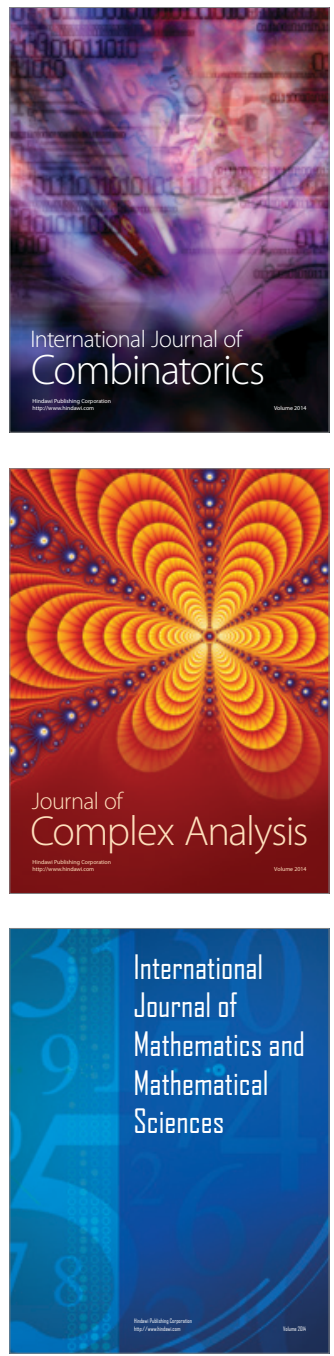
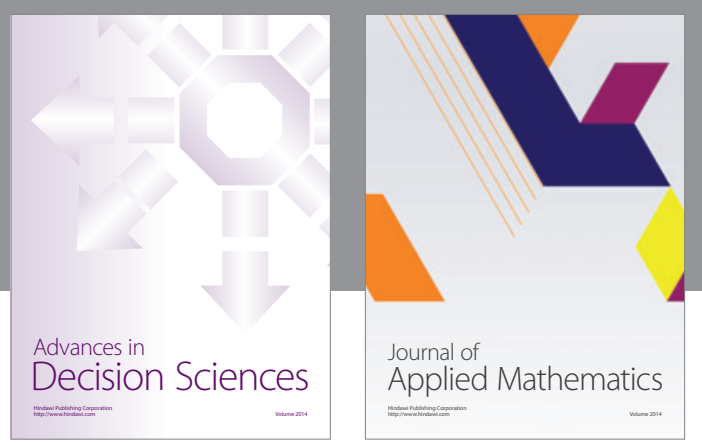

Algebra

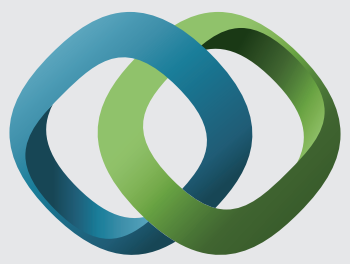

\section{Hindawi}

Submit your manuscripts at

http://www.hindawi.com
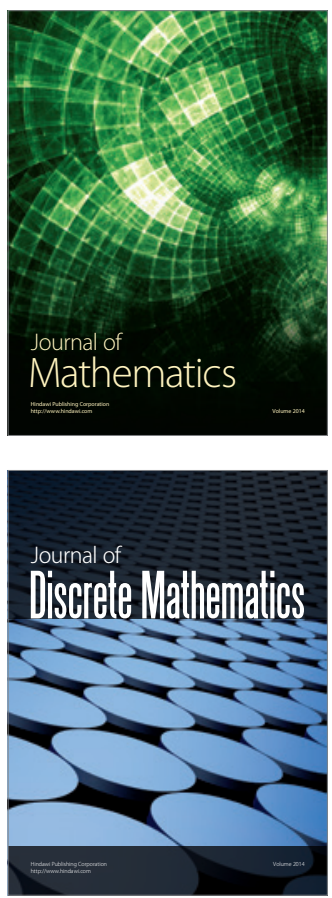

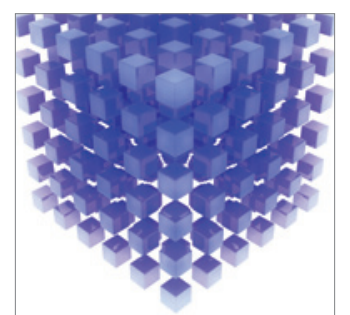

Mathematical Problems in Engineering
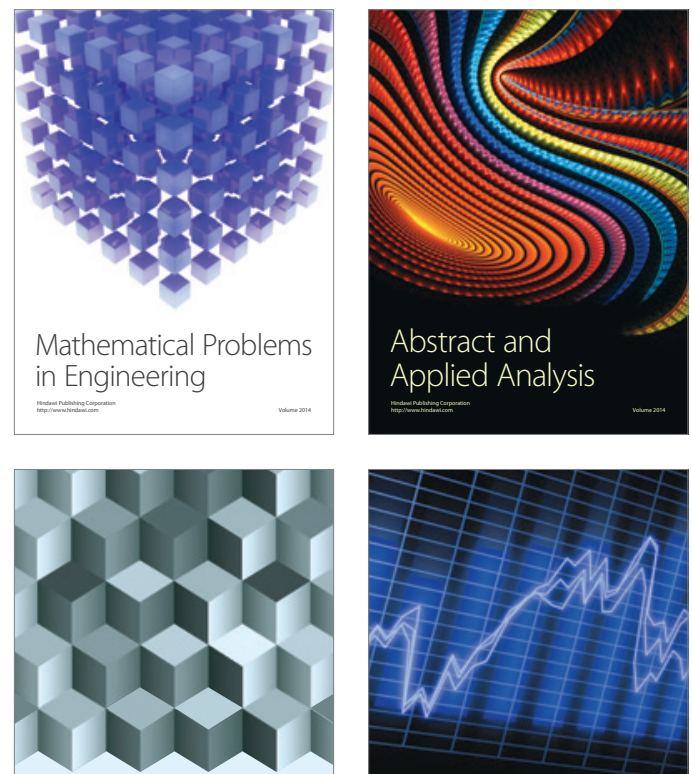

Journal of

Function Spaces

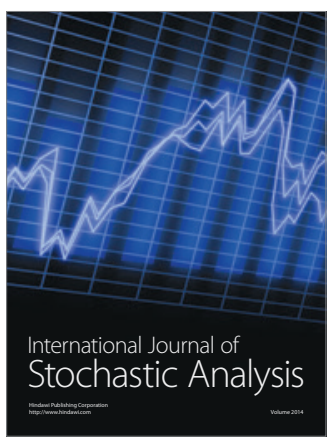

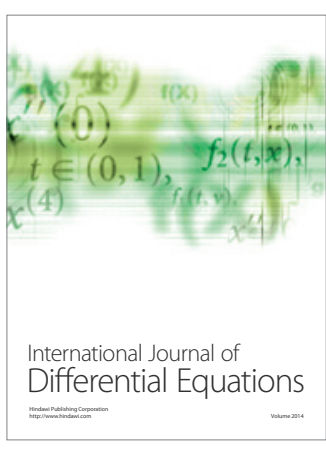
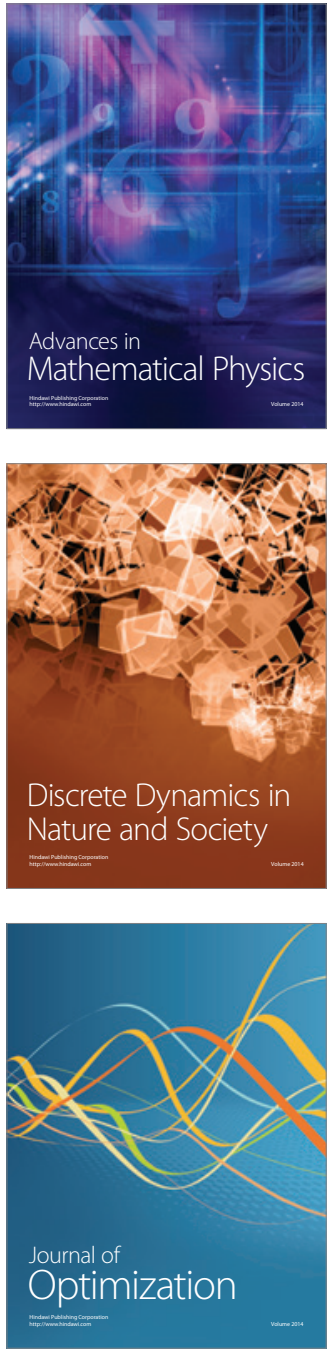